\title{
A Behavior-Analytic Approach to Antivaccination Practices
}

\author{
Sumah V. Confer ${ }^{1} \cdot$ James W. Diller ${ }^{1}$ (D) Jeffrey S. Danforth ${ }^{1}$
}

Accepted: 12 March 2021 / Published online: 8 June 2021

(C) Association for Behavior Analysis International 2021

\begin{abstract}
In recent years, there has been an increase in outbreaks of diseases that are preventable by vaccination. As vaccination involves behavior, behavior analysts are uniquely positioned to contribute solutions to this socially significant problem. The present article explores a behavior-analytic approach to understanding the function of the behavior of both people who have their children vaccinated and those who do not have their children vaccinated, and potential interventions to increase vaccination rates. An introduction to the problem is followed by a brief history of the antivaccination movement. In our analysis, a failure to vaccinate is conceptualized as a noncompliance response (i.e., medical nonadherence), and conditions giving rise to that noncompliance are evaluated. In this process, the roles of avoidance, the functional-altering impact of rule-governed behavior, relational frames, and countercontrol are considered. Potential solutions informed by applied behavior-analytic literature, including contingency management and behavioral safety, are discussed.
\end{abstract}

Keywords Behavior analysis $\cdot$ Culture $\cdot$ Disease $\cdot$ Rules $\cdot$ Vaccination

Vaccination involves the controlled delivery of infectious agents to promote the response of the immune system without direct exposure to a disease. The vaccination of large groups of people reduces the risk of infection of any particular member of that group, in a phenomenon known as herd immunity (Fine et al., 2011). The protection provided by high rates of immunization is important to reduce rates of infection, especially among individuals who are unable to be vaccinated (e.g., very young children, immunocompromised people). Indeed, the World Health Organization recently dubbed the hesitancy surrounding vaccination a top threat to global health (Scheres \& Kuszewski, 2019).

James W. Diller

dillerj@easternct.edu

1 Eastern Connecticut State University, Willimantic, CT, USA 
According to the Centers for Disease Control and Prevention (CDC, 2018), a single dose of the measles vaccine, also known as the MMR (measles, mumps, and rubella) vaccine, is $93 \%$ effective at preventing the disease, which is lethal in approximately $15 \%$ of cases (CDC, 2019). A second dose raises the effectiveness to $97 \%$. Although measles was almost eradicated after the MMR vaccine was developed in 1963, reported cases have now reached the highest peak in 25 years (CDC, 2019). This exemplifies how a potentially deadly disease such as measles can reemerge despite the availability of a preventative vaccine. The measles reemergence did not occur by chance, but rather is the result of a shift in human behavior.

The return of measles may be a product of the antivaccination movement, which propagates the theory that vaccines are dangerous and unnecessary and can lead to an array of neurological and physical ailments (Boulanger, 2017). A growing population of followers, sometimes referred to as "antivaxxers," subscribe to this movement even though it lacks scientific evidence to support its claims. Subjecting oneself, one's offspring, or the community to preventable diseases creates serious public-health problems and is therefore undesirable.

The attitudes of individuals who engage in antivaccination behaviors (i.e., failing to vaccinate their own children or advocating that others do not vaccinate) have been examined in the field of psychology. For example, Hornsey et al. (2018) found that opposition to vaccination was highest among people who also had high levels of conspiratorial thinking. Although this may be important in understanding antivaccination behaviors, a behavior-analytic approach to explain the contingencies controlling the behaviors that compose the antivaccination movement may offer functional analyses that describe controlling variables that may be modified in interventions to increase vaccination. The purpose of this article is to explore a behavioranalytic approach to understanding the behavior of people who do or do not have their children vaccinated, and to provide potential strategies to increase vaccination that are modeled on empirically validated interventions.

\section{A Brief History of Vaccines and Antivaccination Ideology}

Immunizations were first evident in the use of smallpox inoculations in China as early as 1567 (Boylston, 2012). In the late 1700s, Dr. Edward Jenner of the United Kingdom created a smallpox vaccine that contained cowpox in place of smallpox (Godfrey, 1881). Although this was safer than previous methods of injecting smallpox materials to inoculate against the disease, skepticism arose because the population did not yet fully trust doctors, medicine, or the general safety of vaccines. Yet, due to the effectiveness of the vaccine, England enacted the Vaccination Act of 1853 (Brunton, 2008). This act required vaccinations for children up to 3 months old and included penalties for noncompliance. The enforcement of this act resulted in the formation of the Anti-Vaccination League, and prominent supporters such as William Tebb became known as antivaccinationists. Tebb traveled to the United States in 1879, where he disseminated the beliefs of the Anti-Vaccination League and influenced the creation of the Anti-Vaccination Society of America (Wolfe \& Sharp, 2002). Six years after Tebb's visit to the United States, an antivaccination demonstration march consisting of approximately 100,000 people took place in Leicester, United Kingdom (Wolfe \& 
Sharp, 2002). By 1898, antivaccinationists achieved a legal victory that would strengthen the roots of the antivaccination movement, when the Vaccination Act of 1853's penalty for noncompliance with vaccination was removed (Wolfe \& Sharp, 2002).

However, a series of events took place over the next 3 decades that would shift the world's focus back to vaccinating. The 1905 U.S. Supreme Court ruling of Jacobson $v$. Massachusetts declared that states could protect the public by instilling compulsory laws that would require vaccination against communicable diseases (Mariner et al., 2005). By the 1940s, there were vaccines for pertussis, tetanus, diphtheria, and smallpox, and vaccines for diseases like polio and typhoid were available by the late 1950s (Children's Hospital of Philadelphia, 2019). Hotez (2018) noted that the 14 diseases currently targeted for childhood vaccinations in the United States have essentially disappeared due to the efficacy of the vaccines that have been developed.

In 1998, Andrew Wakefield became the catalyst of the modern-day antivaccination movement, when he fabricated a link between vaccinations and autism in a paper that was published in The Lancet, which was subsequently retracted due to ethical concerns (The Editors of the Lancet, 2010; Eggerston, 2010). As Wakefield's findings quickly garnered international media attention and rose in popularity, the percentage of children who received a measles diagnosis also rose (CDC, 2015), perhaps signaling that Wakefield's findings and subsequent dissemination of these findings may have influenced people's vaccination choices.

In 2000, there were only 86 confirmed cases of measles within the U.S. population, marking a record low (CDC, 2002). However, in 2013 there were 159 cases of measles reported, and by 2014, there were 668 cases reported in the United States (CDC, 2018). In the decade after Wakefield's initial publication, numerous papers would refute his claims, and the findings were debunked in the scientific literature. Gerber and Offit (2009), for example, reviewed 20 epidemiological studies and concluded that there is no evidence of a causal relationship between thimerosal, an organic compound containing mercury that had been used as a preservative in vaccines, or the MMR vaccine and autism. Twelve years after the paper's release, Wakefield's medical license was revoked, most of his coauthors had renounced their authorship of the paper, and it was retracted by the prestigious medical journal in which it was published. Despite that retraction, the rates of preventable diseases have continued to rise. In what follows, we will evaluate possible behavioral mechanisms that might influence an individual's choice to have their child vaccinated.

\section{Why or Why Not Vaccinate: A Behavioral Account}

The conceptualization of a failure to vaccinate is complex due to the broad array of antecedent events occurring before the choice to participate in vaccination, which is a part of a medical regime. Following a medical regime, such as receiving scheduled vaccinations, is described as the behavioral process of adherence (Alloway, 2016, slide 6). Therefore, failure to complete vaccinations is nonadherence to a medical regime. This nonadherence can be intentional or unintentional (Alloway, 2016, slide 7). Actively choosing not to vaccinate despite the ability to do so is an example of intentional nonadherence, whereas unintentional nonadherence could involve forgetfulness or a lack of access to care. The failure to vaccinate is conceptualized as both 
intentional and unintentional nonadherence to a medical regime attributed to a range of motivating operations and setting events, including antivaccination demonstrations, peer influence, personal and religious beliefs, and socioeconomic status.

It is important to differentiate antivaxxers from those who are vaccine hesitant or vaccine refusers. Active engagement in opposition to vaccination, such as campaigns and efforts to influence vaccine hesitancy and vaccine refusal, is the defining characteristic of antivaxxers (Whitehead et al., 2019). Someone who refuses to vaccinate does not have to be an antivaxxer. Thus, there are complexities in understanding nonadherence to a vaccination regime. Antivaccination, vaccine hesitancy, and vaccine refusal cannot be explained based on a single antecedent or motivating operation because numerous motivating operations, setting events, and contextual factors influence variability in vaccination practices.

\section{Avoidance}

A cursory analysis is that a parent has their child vaccinated so the child avoids contracting measles, mumps, or rubella because such an event would be aversive to the parent. However, using avoidance research to understand the vaccinating behavior among parents is inadequate. For example, a parent may be given a warning that if they do not vaccinate their child, their child can develop a deadly disease. Such a warning may be conceptualized as a conditioned establishing operation (Miguel, 2013), as it alters the effectiveness of a vaccination as a reinforcer. If a parent responds to the warning from the community and has their child vaccinated, there is, in fact, no outcome that could be described as a consequence related to receiving the vaccine. That is, nothing happens; failing to contract a disease is not a behavioral event.

If this were true avoidance, it suggests a more distal relationship between the avoidance response and the aversive event that is avoided than is commonly conceptualized. Similarly, in typical avoidance research, the same organism that emits avoidance behavior has directly experienced the aversive stimulus. Most parents state their intention to vaccinate, and then either do or do not have their child vaccinated, well before their children contract preventable infectious diseases.

Interestingly, there are uncontrolled case studies reported in the press that may illustrate the avoidance conditioning processes. For example, Tara Hills, a parent of seven children in a remote area of Ottawa, Ontario, Canada, did not vaccinate her children against pertussis. Also known as whooping cough, pertussis is a severe infectious disease that is life threatening for young children. The first three of Hills's children had received a partial amount of the recommended vaccines, but following media reports, conflicting information, and numerous theories that confused Hills and her husband about the safety of vaccines, their four younger children were not vaccinated. Later, all seven children developed whooping cough, and a measles outbreak occurred nearby. At this point, Hills had directly encountered a situation wherein her children had an infectious and preventable disease that could have been avoided had Hills attended to medical directives encouraging vaccination earlier. Hills subsequently vaccinated all of her children and is now a prominent supporter of vaccination (Bernstein \& Schatz, 2015).

Hills's support of vaccination occurred only after encountering the illness, which could have been prevented with a vaccine. This mother's response to her children 
contracting whooping cough illustrates a contingency-shaped avoidance behavior. For an avoidance response to be contingency shaped, there must have been direct contact with environmental contingencies, as opposed to verbal discriminative stimuli (i.e., rules; Skinner, 1974). In this case, contracting the preventable illnesses created the contingency. However, it is important to consider the unknown number of parents who do encounter these diseases and still do not vaccinate their children. Examples of such contingency-shaped behavior may explain why a parent chooses to vaccinate after failing to so, but such episodes are exceedingly rare and do not explain why most parents who have never encountered such contingencies have their children vaccinated.

The vaccination behavior of most parents is not a result of direct experience with infectious disease but, instead, is driven by other factors. In the absence of direct contact with contingencies, we may conceptualize most parents' vaccinating behavior as rule governed.

\section{Rule-Governed Behavior}

Unlike contingency-shaped behavior, rule-governed behavior does not necessarily involve direct contact with the contingency. Instead, a verbal description of a contingency may evoke a behavior or alter an existing discriminative relation (Schlinger \& Blakely, 1987). "If you vaccinate your child, they will develop autism" and "If you don't vaccinate your child, they may develop measles" are examples of rules that could govern a parent's vaccination behavior. Physicians may present vaccination schedules in terms of rules (e.g., "At 12 months of age, your child should receive the MMR vaccine to prevent this disease"). Other rules and motivating operations develop outside of conversations between parents and their health care providers.

For many people, religious practice and belief generate a wide range of motivating operations that can have a major role in decisions about vaccination, and may be a source of rules. The influence of religious culture on vaccination varies greatly across groups. For example, Catholics partake in vaccination but do not support the development of vaccines from fetal cells and call for alternative research methods (The College of Physicians of Philadelphia, 2018). Muslims intend not to use vaccines containing pork products; however, most vaccinations do not contain these products. Islamic views on vaccination are clear based on the Dakar Declaration on Vaccination (2014), which emphasizes the importance of vaccines, encourages vaccination, and is signed by leaders of the faith. Jewish rabbis also encourage families to vaccinate in order to protect one's life, a commandment in the Torah (Najera, 2018). A common misconception about the role of religion in vaccination decisions is that many religions forbid vaccination. This is not as common as one might believe; in the United States, only the Church of Christ, Scientist, and the Dutch Reformed Church have theological objections to vaccination (Najera, 2018).

Grabenstein (2013) suggested that putatively religious concerns about vaccinations are actually concerns about safety. For instance, one Facebook user commented, "The bible says not to put such things into our bodies it causes disease and destroys the God gland, (our antenna to Gods [sic] intelligence)" (Nay, 2019). Although such a gland does not exist, the author of this post was stating a rule: a verbal description of what is believed will happen after vaccinating a child, or the contingency between a vaccinating response and a putative consequence. In this case, the author wrote that if their child 
is vaccinated, the child's communication or connection with God will be restricted. Although this rule may not be of concern in the general population, for this person, it was apparently sufficient to reduce the value of vaccination.

In general, religion capitalizes on motivating operations to maintain adherence to its rules and practices. If someone within a religion is commanded not to vaccinate, or they experience social pressure from other community members to not vaccinate, they may feel that the potential consequences for vaccinating against their community's wishes are too severe to risk experiencing. Such consequences (real or imagined), such as resentment, shame, or concerns about eternal damnation, may be sufficiently strong to prevent vaccination. The reinforcement of following commands, such as access to God, eternal salvation, and respect from others, is notably powerful. As Diller and Boornazian (2015) noted,

Although heaven and hell cannot directly have effects on an organism (as they are supernatural), they may be treated as ultimate or deferred consequences that derive power from the verbal behavior related to them. The reinforcing potency of such consequences is facilitated by more proximate contingencies upheld by the members of the verbal community (e.g. parents, clergy). (p. 297)

However, despite religions controlling the behavior of members of small sects with powerful consequences and motivating operations, claiming religious exemption from vaccination is still an example of intentional nonadherence to a medical regime.

Unfortunately, there is not one simple rule that governs every antivaccination behavior. In fact, some are quite complex. Rules may be highly idiosyncratic to the individuals who choose to not vaccinate. This characteristic of the rules stated by antivaxxers, combined with underlying complexities (see the Culture and Vaccination section that follows), poses a challenge to promoting behavior change. However, rules offer the benefit of not requiring contact with terrible, preventable diseases.

For understanding vaccinating behavior, there are some benefits of accounts based on rule governance. Rules can simplify a complex contingency, especially one with distal outcomes, making it easier to explain and understand (Skinner, 1974). This makes rules easier and faster to learn compared with behavior that is only shaped by contingencies. This precludes the necessity of childhood illness serving as a consequence that alters the probability of vaccinating behavior, which is a great advantage from a public-health perspective.

\section{Disadvantages of Rules}

One limitation of rule governance is that a rule may not correspond to the actual contingency. For example, the rule "Do not go over the speed limit, or you will get a ticket" is rarely enforced in relation to how many people exceed the limit and how many people receive a ticket. As such, the rule is not very accurate, and the behavior of few drivers is affected by such a rule. In comparison, if a person directly contacted the contingency specified by the rule (i.e., received many speeding tickets), they may be much less likely to speed in the future due to their history of contact with the speedlimiting contingency. With respect to vaccinations, parents do not experience direct contact with the contingency (i.e., a child's illness that could have been prevented by 
vaccination). As such, their behavior is rule governed and may not be as concisely related to the contingencies as parents who have experienced direct contact with the illness (Skinner, 1974). This is only one example of a contingency that may be present.

\section{Rules May Prevent Contact With Contingencies}

Reconsider the scenario where the parent vaccinated her children only after she came into contact with the actual contingency in which not vaccinating her children against whooping cough resulted in them developing the illness. This direct contact acted as a stronger agent of change than the rule given to govern the behavior of not vaccinating. Rules such as "If you vaccinate your child, they will develop autism" describe enough of a contingency between behavior and consequence that some parents choose not to vaccinate. This is simply due to learning history. For example, people who report needles and blood as being highly disgusting, which can be inferred as being a result of learning history, are often among those who hold antivaccination attitudes (Hornsey et al., 2018). These stated consequences can act as reasons why a person may believe the warnings given (Skinner, 1974). Simply stated, when antivaccination behaviors are rule governed, parents have no reason to change their behavior unless they have come into contact with a stronger competing contingency.

In 1980, Skinner described the difference between contingency-shaped and rulegoverned behavior in the context of whether or not parents have their children vaccinated as follows:

The failure of many parents to have their children vaccinated shows the differences between rules and contingencies. In the days when parents saw many children crippled by polio, they rushed for the new vaccine for their own children. Now that there are few cases, it is hard to get them to have their children vaccinated. Contingencies grow weak, and rules are weak. (p. 87)

Skinner was prescient; the reduced prevalence of measles, mumps, and rubella represents a change in the actual contingency, and the rate of vaccination has correspondingly decreased.

Research evaluating the difference between rule-governed and contingency-shaped behavior has shown that behavior under the control of rules may be less sensitive to environmental changes than contingency-shaped behavior. Early human operant research illustrates that both high-rate (Matthews et al., 1977) and low-rate (Shimoff et al., 1981) responding maintained by instructions may be less sensitive to changes in contingencies, particularly when instructed responding prevents contact with contingencies (Galizio, 1979). Although rule-governed behavior may be easier to acquire than contingency-shaped behavior, rules may not always produce effective outcomes when contingencies change. Often, rules evoke behavior that is limited in range, especially when a rule describes a discrete response. But, if there is variability in responding, whether it be instructed or contingency shaped, that variability allows people to contact a wider range of potential outcomes, resulting in responding that is more sensitive to the actual contingency and thus more efficient - the highest rate of reinforcement with the least amount of effort (Joyce \& Chase, 1990). Unfortunately, in the context of vaccinating behavior, there is a remarkably limited range of possible 
responses. Parents either tend to vaccinate or not, rarely coming into contact with consequences of varying outcomes. The result is that vaccinating behavior governed by rules tends to be very steady, changing little, even as contingencies shift. However, there are many motivating operations and setting events that create these contingencies.

Strategies to evoke vaccination behavior that include rules or instructions might be less effective if parents are not contacting either their own children with preventable diseases or state-controlled sanctions, or if motivating operations from social communities (e.g., religious, online) are strong. In a sense, the very effectiveness of vaccines may have served as an establishing operation (e.g., Michael, 1983). Today, parents rarely see children with the diseases that vaccines prevent. As such, the reinforcing value of vaccines to prevent these diseases is reduced, and the probability of behavior that leads to vaccinations (taking one's child to the doctor for vaccination) is weakened among some individuals. This is not applicable across all individuals because some may be less sensitive to rules than others. For example, Hornsey et al. (2018) found that an individualistic worldview was a common trait among people who were considered to have high levels of antivaccination attitudes. These individuals are disproportionately reinforced by events that meet their own individual needs as opposed to events that meet the needs of a larger group or culture; therefore, their sensitivity to rules such as "vaccinating to support herd immunity" will likely be weaker. As Skinner (1980) asked, "To what extent is one's own conduct changed upon seeing another person punished (by authorities or by nature, as in a car accident or in a crippling disease)? Is nature's capital punishment a deterrent?" (p. 87).

\section{Relational Frame Theory}

Whereas the present analysis has primarily focused on rule governance as a method to understand antivaccination practices, relational frame theory also could be applied to better understand this phenomenon. The wide array of interpersonal and cultural antivaccine messages to which some parents are exposed suggests that numerous unrelated messages are being experienced, but advances in relational framing provide a prism that might connect these seemingly disparate events. Relational frame theory emerged from work on rule-governed behavior (Skinner, 1969) and stimulus equivalence (Sidman \& Tailby, 1982) with a focus on language (Barnes-Holmes et al., 2018). Parents are exposed to a seemingly endless variety of ostensibly unrelated antecedentconsequent relations that decrease the probability of vaccinating a child. Parents may be exposed to events indicating that vaccines are undesirable for religious reasons, because they represent governmental interference or a loss of personal liberty, because they cause autism or other diseases, because they lead to social or familial ridicule, because they represent an antimachismo veneer, because science and the government are untrustworthy, and so on. These disparate events are presented via television, radio, the internet, and interactions with commentators, newscasters, celebrities, friends, and family. We suggest that these disparate events have come to participate in a common relational frame.

We are judgmental in the sense that we consider it "desirable" for both individuals and the culture that children be vaccinated. Nonetheless, we acknowledge that the stimuli that compose the relational frame are not necessarily logical (Hayes, 1991) in terms of what is "desirable." Hayes (2004) wrote that "relational learning will transfer 
to events that are not necessarily related formally but rather are related on the basis of these arbitrary cues ('arbitrary' in this context means 'by social whim or convention')" (p. 648). The relational antivaccine responding (including emotions and cognitions) to a diverse set of stimuli emerges from a conditioning history, leading to a transfer of function between the elements of the stimulus class, such that, for example, hearing a radio announcer scream that vaccines limit personal freedom and watching a family member berate someone for vaccinating a child come to serve the same function - a reduced probability of vaccinating - even if the parent never had their personal freedoms limited or were never berated. Language allows the parent to relate these events that are under arbitrary textual control, wherein freedom, religion, politics, love for a child, and so forth are nonetheless formally related via "transformation of stimulus functions among related stimuli" (Hayes, 2004, p. 648). The different stimuli "share some of the functions of other stimuli in the network by virtue of their associations.... Any one of these stimuli, if experienced, could thus deliver some stimulus functions of their coordinated stimuli" (Blackledge, 2003, p. 423). As such, the combination of experiences decreases the probability of a parent vaccinating their child.

\section{Culture and Vaccination}

Skinner (1981) noted that behavior is selected by the evolutionary history of the species, the learning history of the individual, and the effects of cultural practices. Choosing to not vaccinate is a behavior that emerges, like all behavior, from knowable events in the historical and contemporary environment. In this section, we briefly describe some factors that might contribute to choosing to not vaccinate.

The choice to vaccinate children (or not) emerges within a cultural setting. Such practices are maintained by "the special contingencies maintained by an evolved social environment" (Skinner, 1981, p. 502). Family and community members provide opinions about vaccination. These opinions may function as rules, signaling the availability of reinforcement for compliance with them, or punishment for failing to follow them. In a verbal community where antivaccination rules are common, choosing to vaccinate a child may be met with criticism, ridicule, or ostracism. The interplay between the behavior of the individual making a decision about vaccination and the behavior of the group surrounding them may represent what Glenn (2003) referred to as "interlocking behavioral contingencies," which may, themselves, replicate and influence the evolutionary course of the culture.

Skinner (1981) suggested that "it is the effect on the group, not the reinforcing contingencies for individual members, which is responsible for the evolution of the culture" (p. 503). Thus, if choosing to not vaccinate has not been met with ill effects (i.e., contraction of preventable disease), this response might persist within that particular verbal community, and such a group would be more likely to generate contingencies to further propagate these behaviors.

Within the cultural context described previously, personal liberties may be more highly valued than the needs of the larger group. Thus, complying with out-group rules associated with vaccination (as delivered by a doctor or community official) may be aversive for members of the antivaccination community. Thus, refusing to vaccinate could represent a form of countercontrol (i.e., efforts to escape or avoid aversive control 
by individuals under aversive control; Sidman, 1989/2000). The antivaccination movement is an example of passive resistance, a form of countercontrol. Members of this movement are not typically violent; however, they do resist the authority of individuals who insist on vaccination.

Active antivaxxer demonstrations also create social influence over vaccination decisions, especially through peers and media. Johnson et al., (2020) suggested that antivaccination Facebook groups outnumber provaccination groups, and that despite a lower following per group, posts from antivaccination groups are shared more often in other Facebook groups between peers. Essentially, these groups, and by extension the information they disseminate, are shared and discussed more frequently than the information disseminated by provaccination groups. Warnings about vaccines created by activists online are often shifted to live protests, such as the current protests against future COVID-19 vaccines. When someone chooses not to vaccinate under these motivating operations, they will receive strong praise from fellow antivaccination peers online, a valuable reinforcement in the modern age.

Antivaxxer protests typically incorporate political and personal belief systems, which may function as motivating operations that influence vaccination decisions, such as distrust in the safety of vaccines. Claiming political or personal belief exemptions, regardless of whether the person is an antivaxxer, vaccine hesitant, or vaccine refuser, is a further example of intentional nonadherence to a medical regime. Here it is important to note the growing distrust in science and antiscience attitudes. Some parents who delay or refuse vaccination regimes can be swayed into adherence by medical professionals who offer assurance and further information (Gust et al., 2008). However, in one study, approximately $25 \%$ of parents indicated that they place their trust in the vaccine-safety information given by celebrities (Freed et al., 2011). For example, Jessica Biel and Jenny McCarthy are well-known celebrity mothers who have openly discussed their opposition to vaccination (Dickson, 2019). In this scenario, and after choosing not to vaccinate, parents could receive reinforcement by the feeling of "doing the right thing" or via peers and idols, such as celebrities who oppose vaccination.

Access to vaccinations, based on socioeconomic status, may be another cultural factor that influences vaccination behaviors. Due to a lack of access, funds, or resources, some people may not be able to vaccinate themselves or their children. For example, in 2013, a mother was not able to vaccinate her child because of an unpaid pediatrician bill (Parasidis \& Opel, 2017). Due to the outstanding bill, the pediatrician would not see her children until it was paid. With the motivating operation present in this example (financial strains), the consequence of vaccinating would be not having a sustainable amount of funds remaining. This is a stressful and problematic consequence that many families wish to avoid. Instead, not vaccinating, and therefore being able to afford general necessities, becomes more reinforcing. In another example, a parent with severe back pain and little social support fell behind on her child's vaccination schedule (Parasidis \& Opel, 2017). Due to the pain and lack of support, staying at home was easier than going to a doctor's office. The parents in these examples could not vaccinate due to issues that can reasonably be described as beyond their control, and both parents in these examples expressed that they would have vaccinated if they had the ability to do so (Parasidis \& Opel, 2017). These are both examples of unintentional nonadherence to a medical regime. 
When choosing to not vaccinate is viewed as a part of a cultural-behavioral process, it may increase the compassion felt toward the people making this choice, because they could make no other choice. As Chiesa (2003) noted, "determinism encourages respect for persons and compassion in the treatment of those whose behavior is less than desirable" (p. 244). From the perspective of the broader culture, engaging in practices that reintroduce preventable disease, particularly in children or individuals who are immunocompromised, is undesirable. Thus, behavior analysts should approach this problem with a combination of compassion and evidence-based interventions.

\section{Behavior-Analytic Proposals to Increase the Probability of Vaccinating Responses}

\section{Modifying Motivating Operations}

Making an effort to modify motivating operations is one solution. For example, personal, religious, and antiscience-related motivating operations can be countered by making other valuable reinforcers contingent on vaccination. Although all states currently require vaccinations for children to attend school, exemptions for personal beliefs are possible to obtain. In states where those exemptions are easier to acquire, there have been increasing trends in obtaining them (Omer et al., 2012). Thus, increasing the difficulty of receiving vaccination exemptions might increase the probability that parents will vaccinate their children. Of course, such policy changes could not be achieved by behavior analysts alone, but would require partnership with governmental agents. Modifying the motivating operations of antiscience attitudes lies partly in education and partly in, again, making it more difficult to receive an exemption. One example that combines both of these factors is in Washington State. People applying for an exemption must attend a meeting with a physician to discuss scientific vaccination information and decisions (Opel \& Diekema, 2012).

Another way to modify motivating operations related to religious belief would be to ask more leaders within religions to release documents such as the World Health Organization (2014), which explains the importance of vaccines and clarifies that vaccination is permissible within Islam. Furthermore, this document underscores the moral imperative for parents to provide adequate health care, including vaccinations. Because most religious communities in the United States are not against vaccinations (Grabenstein, 2013), having more well-publicized statements in support of vaccinations from religious leaders could bolster vaccinations in their congregations.

Beyond modifying motivating operations related to intentional nonadherence, there must be an effort to decrease unintentional nonadherence to vaccination regimes. In instances where parents cannot afford vaccines, Opel and Diekema (2012) asked that public vaccination funding programs be created to assist families that cannot afford vaccines. Another modification that may help families with financial struggles while also offering incentives to others is being implemented in Australia, where a public policy is in place that incentivizes vaccines by offering lower insurance rates and tax rebates in exchange for vaccination (Opel \& Diekema, 2012). 


\section{Rule-Governed Behavior}

When rules are effective, "they are effective only because special reinforcement has been made contingent upon them. Governments, for example, do not trust the natural advantages of obeying the law to ensure obedience" (Skinner, 1969, p. 148). To promote vaccination-related behavior among individuals who might otherwise resist it, programming social reinforcement or punishment might be necessary. This is the manner in which the U.S. government altered the probability of smoking. As Skinner (1969) noted,

A formal statement of contingencies (Cigarette smoking causes lung cancer) needs the support of carefully engineered aversive stimuli involving sanctions quite possibly unrelated to the consequences of smoking. For example, smoking may be classified as shameful, illegal, or sinful and punished by appropriate agencies. (pp. 149-150)

Based on the recent resurgence of preventable (or, indeed, previously eradicated) disease, it seems that sufficient aversive stimuli are not presently in place to compel the decision to vaccinate. This is most currently demonstrated by the rollout of a COVID-19 vaccine. The COVID-19 pandemic has had profound effects on all areas of life across the globe. However, despite people coming into direct contact with the effects of COVID-19, vaccine hesitancy and refusal toward a COVID-19 vaccination are largely present in public concerns. These concerns range from reasonable, such as weighing the safety risks of a newly developed vaccine, to questionable, such as antivaxxer claims that the vaccine will contain a tracking chip to be used by the government as surveillance (Putterman, 2020). These claims and concerns have quickly spread. In an ABC News poll, a quarter of responders said they would not receive a COVID-19 vaccine, and a third of responders to a CNN poll said they would not receive one (Dreisbach, 2020). According to Dr. Anthony Fauci, the top infectious disease expert in the United States, a COVID-19 vaccine would likely be ineffective in creating herd immunity if only two thirds of the population received it (Dreisbach, 2020). Now is a crucial time to modify contingencies and develop methods that will encourage vaccination.

\section{Why Rules Might Be a Good Idea}

Skinner elucidated differences between rule-governed and contingency-shaped behavior, and the differences described suggest that rule governance could be useful for promoting cultural change. For example, rules "will evoke behavior when reinforcing consequences are very rare and contingency-shaped behavior therefore unlikely. . . . Long deferred consequences, ineffective in shaping behavior, may also lead to useful rules" (Skinner, 1969, p. 168). In a culture approaching herd immunity via vaccination, mumps is rare. When they do emerge, it is long after the act of refusing vaccination. Temporally distal consequences are weaker than immediate consequences (e.g., Dews, 1960; Skinner, 1938), so it may be helpful to construct rules to promote vaccinating responses: "Rules tend to bring remote consequences into play; without rules only immediate consequences affect behavior" (Skinner, 1969, p. 169). Certainly, it is best if children do not ever get mumps or other diseases to make the rules more powerful. 
The publication and dissemination of Wakefield's findings established a rule in the zeitgeist: Vaccines cause autism. With repetition by media outlets and celebrities, seemingly plausible claims can become more likely to be believed (Hasher et al., 1977). This effect can be reduced by focusing on the accuracy of the claim at the initial exposure to the idea (Brashier et al., 2020). When one is told that a claim is true and then later learns that it is false, it can still influence judgments about causality $(\mathrm{H}$. M. Johnson \& Seifert, 1994). H. M. Johnson and Seifert (1994) found that receiving corrected information is not sufficient to change judgments about the truth of the initial claim. Instead, plausible causal alternatives are necessary. Thus, broad campaigns to increase critical evaluation of false claims, coupled with the delivery of good information about the current understanding of medical science, may help to combat antivaccination behavior. Analysis of such campaigns via relational frame theory may be warranted in future research.

\section{Prompting and Effort Reduction}

Researchers working in public health have found that prompts, in the form of postcards, signed letters, and text messages, increase the probability that individuals will be vaccinated. For instance, Larson et al. (1982) found that postcards containing information about susceptibility to a disease, severity of the disease, and risks and benefits of vaccination increased the likelihood that individuals would receive an influenza vaccination, relative to no postcard or a neutral postcard. More recently, Matheson et al. (2014) determined that reminder text messages increased the probability of completing the HPV vaccination series, relative to standard care procedures. These authors also found that reducing the effort required to opt in to the text reminders led to higher rates of completion than a more effortful process.

The notion that lower effort results in greater vaccination adherence is further supported by a study by Dexter et al. (2004). These authors evaluated the likelihood that hospitalized patients would receive vaccinations for pneumonia and influenza. When standing orders were in place (i.e., the vaccination happened in the normal course of care), vaccinations were more likely to be completed than when reminders were provided by physicians. Taken together, these studies indicate that prompting may promote vaccinations, but automatizing them may be more effective. Of course, these efforts may only be effective for individuals who are not vaccine hesitant.

\section{Modeling}

What is to be done? Describe the epidemics which may come if the children are not vaccinated? Show crippled children on TV? In other words, reestablish modelling contingencies? (Skinner, 1980, p. 87)

Modeling the outcomes of the diseases via popular media may have some effect. For instance, telenovelas have been used to change attitudes and intentions about issues such as workplace safety (Castaneda et al., 2013) and kidney disease (Forster et al., 2016). Thus, similar interventions could use culturally relevant media models to 
potentially influence the vaccination behaviors of communities where vaccination is less likely to occur. This model-based approach could be coupled with reinforcement programs, as in contingency management.

\section{Contingency Management}

Contingency management involves the programming of reinforcement for desired behaviors. This type of intervention has been used to improve a variety of healthrelated behaviors, including drug abstinence (e.g., Stitzer \& Petry, 2006) and exercise completion (e.g., Kurti \& Dallery, 2013). In these applications, participants in programs generally receive financial incentives (small payments or vouchers) for completing the desired response. This approach could be applied to the successful delivery of vaccinations across the recommended childhood schedule. If parents are hesitant to have their children vaccinated, component steps (e.g., seeking a pediatrician, attending regular appointments) could be targeted.

One other intervention model to promote vaccines could come from research promoting healthy food acceptance by children (e.g., Horne et al., 2009; Lowe et al., 2004). In the Food Dudes intervention, rules related to consuming healthy foods are provided, healthy food choices are modeled, and reinforcers are programmed for consumption of healthy foods. A similar intervention strategy could be developed for parents with antivaccination attitudes. Such an intervention could include the modeling of statements about how vaccines are safe and effective, and programmed reinforcement for repeating these statements or having a child vaccinated. The impact of such an intervention could be maximized if such modeling occurred in mass-media outlets. Indeed, there is a growing line of research supporting the utility of "entertainmenteducation" interventions to improve health outcomes (e.g., Castaneda et al., 2013), and vaccination is a logical target for this type of work.

Programming immediate consequences following vaccination may enhance the effectiveness of entertainment-education interventions. A simple and effective way to potentially provide reinforcement to parents who vaccinate is a sticker that says "I vaccinated." The popular "I voted" stickers have become a staple of election days. Using an "I vaccinated" sticker as a reinforcer may attract positive social attention, other secondary reinforcements, and it may act as a visual prompt for other parents. An interesting finding, discussed by Nelson (2016), was that of Facebook's "I voted" sticker initiative, which allowed users to post a digital sticker on their Facebook page. The feature also allowed them to see a list of friends that voted too. However, another group of users only got the sticker option, and some did not get access to the feature at all. As a result of having access to both the sticker feature and the list of fellow voters, there was an increase in voting by roughly 340,000 people, according to Facebook. Applying a similar model for vaccinations may be a cost-effective intervention to increase vaccination rates and potentially counter the antivaccination rhetoric on social media.

\section{Building Compassion}

The medical professionals who are directly engaged in the work of vaccinating children play a vital role in solutions to these problems. It is possible that some medical 
professionals may be intensifying countercontrol by employing aversive strategies (e.g., denying health care unless vaccinations have been administered). Building relationships of trust between the families who are not vaccinating and the medical professionals who serve them may be a necessary component to increase vaccination rates.

Within the behavior-analytic literature, there has been a call for building compassion to solve problems. For instance, Biglan (2015) suggested that creating communities where needs are met and people feel safe and valued leads to solutions to a variety of behavioral problems. Geller (2012) found that when drivers were asked to buckle up by a person holding a sign that read "please buckle up, I care," they were more likely to do so than when they saw a "click it or ticket" sign. The equivalent to "click it or ticket" in this case would be "vaccinate or contract a disease." Therefore, a doctor may be a more successful change agent by "actively caring for people" (Geller, 2012) rather than by relying on threats or aversive prompts.

Here again, the deterministic approach has the potential to yield compassion. People who make the choice to vaccinate, or not, have reasons for doing so, based on their learning history and contemporary environment. And, given their individual circumstances, there can be no other choice made. Thus, by focusing on modifications to the environment and by building additional opportunities for learning, behavior analysts may be able to help achieve a solution to this problem.

\section{Conclusion}

Vaccinations have the potential to eradicate certain infectious diseases, improving public health and the overall standard of living around the world. The refusal to follow vaccination programs is a behavioral problem that is subject to a variety of motivating operations and social reinforcement processes. Modifying this behavior will require change on governmental, social, and provider levels. At the basic level, vaccines should be affordable and accessible, and exemptions lacking medical necessity should be made difficult to receive. Of course, each of the proposed solutions invites further empirical and conceptual development. There is much work yet to be done in this area, and behavior analysts, partnering with other experts, could play a major role in solving this socially significant problem.

Behavior analysts excel at modifying behavior, and the field has several powerful tools available to facilitate behavior change. The application of a behavior-analytic problem conceptualization to this issue may allow for a better understanding of the processes responsible for vaccination behavior. Furthermore, this conceptualization allows for the pursuit of solutions that are both conceptually systematic and capable of solving an important social problem. Further exploration of the ideas presented here would help behavior analysts develop solutions relevant to a broader swath of the population, potentially leading to more mainstream relevance (e.g., Friman, 2010).

\section{Declarations}

Conflict of interest The authors declare no conflicts of interest. 


\section{References}

Alloway, R. (2016). Non-adherence definitions - monitoring - preventions/maintenance [PowerPoint presentation]. U.S. Food and Drug Administration. https://www.fda.gov/media/104649/download

Barnes-Holmes, D., Finn, M., McEnteggart, C., \& Barnes-Holmes, Y. (2018). Derived stimulus relations and their role in a behavior analytic account of human language and cognition. Perspectives on Behavior Science, 41, 155-173. https://doi.org/10.1007/s40614-017-0124-7.

Bernstein, L., \& Schatz, R. (2015). Anti-vax mom changes her tune as all 7 of her children come down with whooping cough. The Washington Post. https:/www.washingtonpost.com/news/to-your-health/wp/2015/ 04/14/anti-vax-mom-changes-her-tune-when-all-7-of-her-children-come-down-withwhooping-cough/.

Biglan, A. (2015). The nurture effect. New Harbinger Publications.

Blackledge, J. T. (2003). An introduction to relational frame theory: Basics and applications. The Behavior Analyst Today, 3, 421-433. https://doi.org/10.1037/h0099997.

Boulanger, A. (2017). Anti vaxxers: Understanding opposition to vaccines. Healthline. https://www. healthline.com/health/vaccinations/opposition

Boylston, A. (2012). The origins of inoculation. Journal of the Royal Society of Medicine, 105(7), 309-313. https://doi.org/10.1258/jrsm.2012.12k044.

Brashier, N. M., Eliseev, E. D., \& Marsh, E. J. (2020). An initial accuracy focus prevents illusory truth. Cognition 194. https://doi.org/10.1016/j.cognition.2019.104054.

Brunton, D. (2008). Compulsory vaccination and divisions among practitioners. In The politics of vaccination: Practice and policy in England, Wales, Ireland, and Scotland, 1800-1874 (pp. 39-53). Boydell \& Brewer, University of Rochester Press.

Castaneda, D. E., Organista, K. C., Rodriguez, L., \& Check, P. (2013). Evaluating an entertainment-education telenovela to promote workplace safety. SAGE Open, 3(3), 1-12. https://doi.org/10.1177/ 2158244013500284.

Centers for Disease Control and Prevention. (2002). Measles-United States, 2000. https://www.cdc.gov/ $\mathrm{mmwr} / \mathrm{preview} / \mathrm{mmwrhtml} / \mathrm{mm} 5106 \mathrm{a} 2 . \mathrm{htm}$

Centers for Disease Control and Prevention. (2015). Epidemiology and prevention of vaccine-preventable diseases (J. Hamborsky, A. Kroger, \& S. Wolfe, Eds., 13th ed.). Public Health Foundation.

Centers for Disease Control and Prevention (2018). Measles and vaccinations. Retrieved April 4, 2019, from https://www.cdc.gov/measles/vaccination.html

Centers for Disease Control and Prevention. (2019). Epidemiology and prevention of vaccine-preventable diseases. https://www.cdc.gov/vaccines/pubs/pinkbook/meas.html

Chiesa, M. (2003). Implications of determinism: Personal responsibility and the value of science. In K. A. Lattal \& P. N. Chase (Eds.), Behavior theory and philosophy (pp. 243-258). Kluwer Academic/Plenum Publishers.

Children's Hospital of Philadelphia (2019). Vaccine history: Vaccine availability timeline. https://www.chop. edu/centers-programs/vaccine-education-center/vaccine-history/vaccine-availability-timeline. Accessed 1 Oct 2019.

Dews, P. B. (1960). Free-operant behavior under conditions of delayed reinforcement. I. CRF-type schedules. Journal of the Experimental Analysis of Behavior, 3(3), 221-234. https://doi.org/10.1901/jeab.1960.3221.

Dexter, P. R., Perkins, S. M., Maharry, K. S., Jones, K., \& McDonald, C. J. (2004). Inpatient computer-based standing orders vs physician reminders to increase influenza and pneumococcal vaccinations rates: A randomized trial. Journal of the American Medical Association, 292(19), 2366-2371. https://doi.org/10. 1001/jama.292.19.2366.

Dickson, E. (2019). A guide to 17 anti-vaccination celebrities. Rolling Stone. https://www.rollingstone.com/ culture/culture-features/celebrities-anti-vaxxers-jessica-biel-847779/

Diller, J. W., \& Boornazian, E. S. (2015). The bonobo and the behaviorist: Frans de Waal's bottom-up morality. The Behavior Analyst, 38(2), 293-307. https://doi.org/10.1007/s40614-015-0031-8.

Dreisbach, E. (2020). Vaccine hesitancy could make it difficult to achieve herd immunity for COVID-19. Healio. https:/www.healio.com/news/infectious-disease/20200714/vaccine-hesitancy-could-make-itdifficult-to-achieve-herd-immunity-for-covid19

The Editors of the Lancet. (2010). Retraction-Ileal-lymphoid-nodular hyperplasia, non-specific colitis, and pervasive developmental disorder in children. The Lancet, 375(9713), 45. https://doi.org/10.1016/S01406736(10)60175-4.

Eggerston, L. (2010). Lancet retracts 12-year-old article linking autism to MMR vaccines. Canadian Medical Association Journal, 182(4), E199-E200. https://doi.org/10.1503/cmaj.109-3179. 
Fine, P., Heames, K., \& Heymann, D. L. (2011). "Herd immunity": A rough guide. Clinical Infectious Diseases, 52(7), 911-916. https://doi.org/10.1093/cid/cir007.

Forster, M., Allem, J., Mendez, N., Qazi, Y., \& Unger, J. B. (2016). Evaluation of a telenovela designed to improve knowledge and behavioral intentions among Hispanic patients with end-stage renal disease in Southern California. Ethnicity \& Health, 21(1), 58-70. https://doi.org/10.1080/13557858.2015.1007119.

Freed, G., Clark, S., Butchart, A., Singer, D., \& Davis, M. (2011). Sources and perceived credibility of vaccine-safety information for parents. Pediatrics, 127(1), S107-S112. https://doi.org/10.1542/peds. 2010-1722P.

Friman, P. C. (2010). Come on in, the water is fine: Achieving mainstream relevance through the integration with primary medical care. The Behavior Analyst, 33(1), 19-36. https://doi.org/10.1007/BF03392201.

Galizio, M. (1979). Contingency-shaped and rule-governed behavior: Instructional control of human loss avoidance. Journal of the Experimental Analysis of Behavior, 31(1), 53-70. https://doi.org/10.1901/jeab. 1979.31-53.

Geller, E. S. (2012). Actively caring for people: Cultivating a culture of compassion. Make-A-Difference.

Gerber, J. S., \& Offit, P. A. (2009). Vaccines and autism: A tale of shifting hypotheses. Clinical Infectious Diseases, 48(4), 456-461. https://doi.org/10.1086/596476.

Glenn, S. S. (2003). Operant contingencies and the origin of culture. In K. A. Lattal \& P. N. Chase (Eds.), Behavior theory and philosophy (pp. 223-242). Kluwer Academic/Plenum Publishers.

Godfrey, E. (1881). Dr. Edward Jenner's discovery of vaccination. Hoeflich \& Senseman.

Grabenstein, J. D. (2013). What the world's religions teach, applied to vaccines and immune globulins. Vaccine, 31(16), 2011-2023. https://doi.org/10.1016/j.vaccine.2013.02.026.

Gust, D., Darling, N., Kennedy, A., \& Schwartz, B. (2008). Parents with doubts about vaccines: Which vaccines and reasons why. Pediatrics, 122(4), 718-725. https://doi.org/10.1542/peds.2007-0538.

Hasher, L., Goldstein, D., \& Toppino, T. (1977). Frequency and the conference of referential validity. Journal of Verbal Learning and Verbal Behavior, 16, 107-112. https://doi.org/10.1016/50022-5371(77)80012-1.

Hayes, S. C. (1991). A relational control theory of stimulus equivalence. In L. J. Hayes \& P. N. Chase (Eds.), Dialogues on verbal behavior (pp. 19-46). Context Press.

Hayes, S. C. (2004). Acceptance and commitment therapy, relational frame theory, and the third wave of behavioral and cognitive therapies. Behavioral Therapy, 35(4), 639-664. https://doi.org/10.1016/S00057894(04)80013-3.

Horne, P. J., Hardman, C. A., Lowe, C. F., Tapper, K., Le Noury, J., Madden, P., Patel, P., \& Doody, M. (2009). Increasing parental provision and children's consumption of lunchbox fruit and vegetables in Ireland: The Food Dudes intervention. European Journal of Clinical Nutrition, 63, 613-618. https://doi. org/10.1038/ejcn.2008.34.

Hornsey, M. J., Harris, E. A., \& Fielding, K. S. (2018). The psychological roots of anti-vaccination attitudes: A 24-nation investigation. Health Psychology, 37(4), 307-315. https://doi.org/10.1037/hea0000586.

Hotez, P. J. (2018). Vaccines did not cause Rachel's autism. Johns Hopkins University Press.

Johnson, H. M., \& Seifert, C. M. (1994). Sources of the continued influence effect: When misinformation in memory affects later inferences. Journal of Experimental Psychology: Learning, Memory, and Cognition, 20(6), 1420-1436. https://doi.org/10.1037/0278-7393.20.6.1420.

Johnson, N., Velásquez, N., Restrepo, N., Leahy, R., Gabriel, N., Oud, S., . . Lupu, Y. (2020). The online competition between pro- and anti-vaccination views. https:/www.nature.com/articles/s41586-020-22811

Joyce, J. H., \& Chase, P. N. (1990). Effects of response variability on the sensitivity of rule-governed behavior. Journal of the Experimental Analysis of Behavior, 54(3), 251-262. https://doi.org/10.1901/ jeab.1990.54-251.

Kurti, A. N., \& Dallery, J. (2013). Internet-based contingency anagement increases walking in sedentary adults. Journal of Applied Behavior Analysis, 46, 568-581. https://doi.org/10.1002/jaba.58.

Larson, E. B., Bergman, J., Heidrich, F., Alvin, B. L., \& Schneeweiss, R. (1982). Do postcard reminders improve influenza vaccination compliance? A prospective trial of different postcard "cues". Medical Care, 20(6), 639-648. https://doi.org/10.1097/00005650-198206000-00010.

Lowe, C. F., Horne, P. J., Tapper, K., Bowdery, M., \& Egerton, C. (2004). Effects of a peer modeling and rewards-based intervention to increase fruit and vegetable consumption in children. European Journal of Clinical Nutrition, 58, 510-522. https://doi.org/10.1038/sj.ejen.1601838.

Mariner, W. K., Annas, G. J., \& Glantz, L. H. (2005). Jacobson v Massachusetts: It's not your great-greatgrandfather's public health law. American Journal of Public Health, 95(4), 581-590. https://doi.org/10. 2105/AJPH.2004.055160. 
Matheson, E. C., Derouin, A., Gagliano, M., Thompson, J. A., \& Blood-Siegfried, J. (2014). Increasing HPV vaccination series completion rates via text message reminders. Journal of Pediatric Health Care, 28(4), e35-e39. https://doi.org/10.1016/j.pedhc.2013.09.001.

Matthews, B. A., Shimoff, E. A., Catania, A. C., \& Sagvolden, T. (1977). Unstructured human responding: senstivity to ratio contingencies. Journal of the Experimental Analysis of Behavior, 27(3), 453-467. https://doi.org/10.1901/jeab.1977.27-453.

Michael, J. (1983). Establishing operations. The Behavior Analyst, 16(2), 191-206. https://doi.org/10.1007/ bf03392623.

Miguel, C. F. (2013). Jack Michael's motivation. The Analysis of Verbal Behavior, 29(1), 3-11. https://doi. org/10.1007/BF03393119.

Najera, R. (2018). Very few religions expressly prohibit vaccination, yet confusion remains. History of Vaccines. https://www.historyofvaccines.org/content/blog/religion-vaccination-confusion

Nay, F. (2019). The Bible says not to put such things into our bodies it causes disease and destroys the God gland, (our antenna to Gods intelligence) [Status update]. Facebook. https://www.facebook.com/groups/ 222916955162773/

Nelson, L. (2016). “I voted” stickers, explained. Vox. https:/www.vox.com/presidential-election/2016/11/8/ 13553112/i-voted-sticker

Omer, S. B., Richards, J. L., Ward, M., \& Bednarczyk, R. A. (2012). Vaccine policies and rates of exemption from immunization, 2005-2011. New England Journal of Medicine, 367, 1170-1171. https://doi.org/10. 1056/NEJMc1209037.

Opel, D., \& Diekema, D. (2012). Find the proper balance between freedom and justice: Why we should not eliminate personal belief exemptions to vaccine mandates. Journal of Health Politics, Policy, and Law, 37(1), 141-147. https://doi.org/10.1215/03616878-1496047.

Parasidis, E., \& Opel, D. (2017). Parental refusal of childhood vaccines and medical neglect laws. American Journal of Public Health, 107(1), 68-71. https://doi.org/10.2105/AJPH.2016.303500.

Putterman, S. (2020). No, the US isn't developing a vaccine or "antivirus" with a chip to track people. Politifact. https://www.politifact.com/factchecks/2020/apr/03/facebook-posts/no-us-isnt-developingvaccine-or-antivirus-chip-tr/

Scheres, J., \& Kuszewski, K. (2019). The ten threats to global health in 2018 and 2019. A welcome and informative communication of WHO to everybody. Zdrowie Publiczne i Zarzadzanie, 17(1), 2-8. https:// doi.org/10.4467/20842627OZ.19.001.11297.

Schlinger, H., \& Blakely, E. (1987). Function-altering effects of contingency-specifying stimuli. The Behavior Analyst, 10, 41-45. https://doi.org/10.1007/BF03392428.

Shimoff, E. A., Catania, A. C., \& Matthews, B. A. (1981). Unstructured human responding: sensitivity of lowrate performance to schedule contingencies. Journal of the Experimental Analysis of Behavior, 36(2), 207-220. https://doi.org/10.1901/jeab.1981.36-207.

Sidman, M. (2000). Coercion and its fallout (rev. ed.). Authors Cooperative. (Original work published 1989)

Sidman, M., \& Tailby, W. (1982). Conditional discrimination vs. matching to sample: An expansion of the testing paradigm. Journal of the Experimental Analysis of Behavior, 37, 5-22. https://doi.org/10.1901/ jeab.1982.37-5.

Skinner, B. F. (1938). Behavior of organisms. Appleton-Century-Crofts.

Skinner, B. F. (1969). Contingencies of reinforcement: A theoretical analysis. Appleton-Century-Crofts.

Skinner, B. F. (1974). About behaviorism. Knopf.

Skinner, B. F. (1980). Notebooks. Prentice Hall.

Skinner, B. F. (1981). Selection by consequences. Science, 213, 501-504.

Stitzer, M., \& Petry, N. (2006). Contingency management for treatment of substance abuse. Annual Review of Clinical Psychology, 2, 411-434. https://doi.org/10.1146/annurev.clinpsy.2.022305.095219.

The College of Physicians of Philadelphia (2018). Human cell strains in vaccine development. https://www. historyofvaccines.org/content/articles/human-cell-strains-vaccine-development. Accessed 1 Oct 2019.

Whitehead, M., Taylor, N., Gough, A., Chambers, D., Jessop, M., \& Hyde, P. (2019). The anti-vax phenomenon. Vet Record, 184(24), 744. https://doi.org/10.1136/vr.14027.

Wolfe, R. M., \& Sharp, L. K. (2002). Anti-vaccinationists past and present. BMJ (Clinical Research Edition), 325(7361), 430-432. https://doi.org/10.1136/bmj.325.7361.430.

World Health Organization. (2014). Dakar Declaration on Vaccination. https://www.afro.who.int/sites/default/ files/2017-09/Religious\%20Leaders\%20Declaration.pdf 